\title{
DIE EMIGRATION ALS STRUKTURPROBLEM DER UNGARISCHEN LITERATURGESCHICHTE
}

\author{
GÁBOR SCHEIN
}

\author{
Eötvös Loránd Universität \\ gaborschein@hotmail.com
}

\begin{abstract}
Die bis heute konsensuale Auffassung der Nationalliteratur in Ungarn wird durch die sprachliche Homogenität bestimmt. Elemente, die eine Provokation für die Homogenität bedeuten, werden entweder in bipolaren Gegensätzen eingeordnet, und dadurch vom Zentrum ferngehalten, oder sie werden vertuscht und ausgeschlossen, wie man es im Falle der auf Fremdsprachen geschriebenen Werke der ungarischen Literatur erfahren kann. Innerhalb der Struktur der ungarischen Nationalliteratur verdient die Emigration eine besondere Aufmerksamkeit, weil sie es notwendig macht, dass wir über die ungarische Literatur in Überlappungen mit anderen Literaturen sprechen. Wenn wir über Überlappungen sprechen, müssen wir in die Problematik auch die Theorie des Transnationalismus mit einbeziehen. In der ungarischen Fachliteratur wird diese Theorie auf die Fälle der geographischen und politischen Grenzübergänge beschränkt, die die mentale Einprägung der Grenzen als Reflexionsobjekte immer voraussetzen. Die Grenzphänomene verbinden sich mit den Vorstellungen der Differenzen, dadurch bleiben sie die Kulminationspunkte der Macht und der Kontrolle. Die Theorie des Transnationalismus kann aber auch dabei helfen, dass die Vorstellungen der Nation aus dem Bereich der homogenisierenden Aspirationen der Macht entfernt werden, damit die Heterogenität des von der Macht territorisierten Raumes, in diesem Fall des literarischen Raumes klar gezeigt werden kann. Die literarischen Räume von Ost-Mittel-Europa hatten schon immer eine mehrsprachige und polikulturelle Ausdehnung, erst nach dem ersten Weltkrieg wurden die Differenzen im Interresse der Homogenisierung der Machtbereichen als Gegensätze instrumentalisiert. Das Aufgreifen des Problems der Emigration macht es möglich, diese räumliche Struktur neuzugestalten, weil die Emigraton nicht einmal als eine periphere Erscheinung am Konzept der ungarischen Nationalliteratur teil hat. Die Emigration kann nicht zuletzt auch die Kräfte des inneren Zusammenhalts im Raum der Nationalliteratur verstärken, denn sie lässt das Aufheben der lokalen Kontexte und die Beseitigung der Differenzen nicht zu, die als Voraussetzungen von Ähnlichkeiten immer ins Spiel gebracht werden. Worüber ich hier spreche, ist eine Hypothese für eine mögliche Selbstkorrektion. Auch diese Hypothese muss ihre Begrifflichkeit der Kritik aussetzen. Wenn aber die Raumstruktur der ungarischen Nationalliteratur als Modell einmal neugestaltet wird, wird die Verwendung des Begriffs der Emigration ihre Gültigkeit verlieren.
\end{abstract}

Schlüsselwörter: Emigration, Emigrantenliteratur, Literaturgeschichte, Nationalismus, Ost-Mittel-Europa 
Die Migration großer Bevölkerungsgruppen erwies sich schon im 20. Jahrhundert als eine der bedeutendsten Ursachen und Konsequenzen globaler wirtschaftlicher und gesellschaftlicher Veränderungen. Keiner der Kontinente blieb davon unberührt. Der Klimawandel, das enorme Anwachsen finanzieller Ungleichheit, die Verbreitung der digitalen Technologien der Gleichzeitigkeit, die Vereinfachung des globalen Reisens und die lokalen Kriege sind die wichtigsten Gründe für die weitere Erhöhung des Migrationspotentials im 21. Jahrhundert. Migrationsbezogene soziale und sprachliche Erfahrungen und deren Wirkung auf die Identifikation der Autoren bzw. der Leser gestalten das literarische Feld weit und tief mit. Hier werde ich mich mit einer bestimmten Form der Migration beschäftigen, die wir Auswanderung oder Emigation nennen. Dies ist die einzige Form der Migration, die sowohl in der Literatur als auch in der Literaturgeschichtsschreibung in Ungarn eine fundamentale Bedeutung gewonnen hat. Die Problematik der Emigration ist in der ungarischen Literatur seit den frühesten Zeiten, in den bekannten, lateinisch geschriebenen Gedichten von Janus Pannonius Laus Pannoniae und Abiens valere jubet sanctos reges, Waradini wahrnehmbar. Das Thema kommt auch in den Anfängen der ungarischsprachigen Literatur, in der Zeit der Reformation oft vor, denn die ersten Reformatoren studierten an deutschen, polnischen und niederländischen Universitäten, und es bleibt auch im frühen 18. Jahrhundert ein zentrales Thema, etwa in den Autobiograhien von János Kemény und Ferenc Rákóczi. Die aus der Perspektive der Moderne sich als normativ erweisende literarische Erlebnisform der Emigration ist im Werk von Kelemen Mikes Törökországi levelek (Briefe aus der Türkei) entstanden. In seinen fiktiven Briefen berichtete der zur Gefolgschaft des Fürsten Ferenc Rákóczi II. gehörende Autor zwischen 1717 und 1758 einer fiktiven Tante über das alltägliche Leben und das Heimweh der ungarischen Emigranten in Rodoschto (türkischer Name: Tekirdağ), wie auch über die Gebräuche und Sitten der Türken. In den späteren Jahrhunderten entstanden in allen ost-mittel-europäischen und west-balkanischen Literaturen mehrsprachige Exiliteraturen. Dazu trugen die sich wiederholenden Auswanderungswellen bei. Es reicht, wenn wir im Falle von Ungarn an die klassischen politischen Auswanderungen in den Jahren von 1848, 1919-20, 1948 und 1956 denken. Die meisten ost-mittel-europäischen Länder erleben gerade wieder einen langen Auswanderungsprozess, der nach 1989 begonnen hat. Diese Auswanderungswelle ist viel komplexer als es die früheren gewesen sind, weil die Migrationsbereitschaft nicht nur in diesem Raum gestiegen ist. Da die Migration die gesellschaftlich-kulturelle Wahrnehmung in den Einwanderungsländern weitgehend beeinflusst, wird sie ihre Spuren in den Kulturen der Auswanderungsländer noch prägnanter hinterlassen.

Die Migration wurde in den letzten Jahrzehnten zu einem allgemeinen Lebensmodell in den europäischen Gesellschaften ${ }^{1}$, d.h. sie gehört praktisch in allen Gesellschaftsschichten bzw. in fast jedem Alter zur normalen Lebenspraxis. Man 
kann sich durchaus die Frage stellen, ob es überhaupt noch adäquat ist, in dieser gegenwärtigen Situation über Emigration zu sprechen. Emigranten sind dem traditionellen Verständnis nach Menschen, die ihre Heimat aus äußerem Zwang verlassen müssen. Ich berufe mich auf die Erfahrungen der Emigranten im 20. Jahrhundert, weil der Kontext der Auswanderung die mentale Wahrnehmung der Migration in Ost-Mittel-Europa immer noch stark beeinflusst, und nicht zuletzt deshalb, weil die Literaturgeschichtsschreibungen in diesem Kulturraum, besonders die ungarische, auf die Erfahrungsformen der Migration im 21. Jahrhundert kaum oder gar nicht reflektieren konnten. Sie sind bis heute blo $\beta$ imstande, auf der Ebene der strukturbildeneden Elemente nur die Emigration wahrzunehmen, und dies oft recht kontrovers und unzureichend.

Bei der Bestimmung des Begriffs „Emigration” zieht man in der Regel drei Merkmale heran: $\mathrm{Ob}$ die Heimat aus politischen oder wirtschaftlichen Gründen veralssen wurde, ob die Flucht legal oder illegal ergriffen wurde, und ob der Emigrant in die alte Heimat ohne strafrechtliche Konsequenzen zurückkehren darf. Nach der strengen Bestimmung von John Neubauer muss man die Person für eine EmigantIn halten, die aus politischen Gründen die alte Heimat illegal verlassen musste, und die in die alte Heimat - bis sich die politischen Verhältnisse grundsätzlich verändern - nicht zurückkehren kann. ${ }^{2}$ In diesem Sinne wären alle diejenigen keine EmigrantInnnen - sondern einfach Auswanderer (expats) - die ins Auswanderungsland ohne strafrechtliche Konsequenzen zurückkehren können. Dieses Kriterium würde ich in der Bestimmung der Emigration nicht gelten lassen, weil die Möglichkeit oder die Unmöglichkeit des Zurückkehrens weitgehend von dem Grund und von den Umständen der Auswanderung abhängig ist. In konkreten Fällen ist es aber gar nicht so leicht zu entscheiden, inwieweit der politische Zwang bzw. die Freiwilligkeit die Flucht herbeigeführt hat, denn mehrere Gründe können zur gleichen Zeit eine Lebensentscheidung bestimmen. Eine EmigrantIn muss ihre Heimat vorherrschend aus politischen Gründen, unter Zwang verlassen, der Auswanderer tut es freiwillig, politisch zwangslos, aus eigener Entscheidung. Auch diese Bestimmung ist indirekt aber sehr stark politisch determiniert. Denn nur im Falle von Diktaturen und Autokratien oder in Bürgerkriegen kann es zu einer derart ernsten Lage kommen, dass sich jemand wegen der politischen Repression oder der direkten Lebensgefahr gezwungen fühlt, die Heimat hinter sich zu lassen. Solche Regime beherrschten den politischen Raum von Ost-Mittel-Europa im 20. Jahrhundert. Kopien dieser Systeme, Reminiszenzen, sind in der Region auch heute durchaus präsent. Deshalb ist es berechtigt, sich in diesem Raum auch im Bezug auf die Literatur mit dem Phänomen der Emigration nachdrücklich zu beschäftigen.

Sollten wir den Unterschied zwischen der Emigration und der Auswanderung auch auf den Bereich der Literatur anwenden, müssten wir die Exil- und die Auswanderungsliteratur kategorisch anders behandeln. In der deutschspra- 
chigen Literaturtheorie geht man bei der Verwendung des Begriffs 'Exilliteratur' bekannter Weise von dem Phänomen der Emigration in dem oben besprochenen Sinn des Wortes aus. Die Bedeutung des Begriffs 'Emigrantenliteratur' ist neutral, er bezieht sich nur auf die Tatsache des Landeswechels. Im Französischen verwendet man die beiden Wörter genau umgekehrt. 'Exiles' ist inhaltlich neutral, 'émigrant' weist auf eine Person, die sich vor der politischen Repression durch Flucht retten muss. So kann die Differenz der beiden Kategorien auch in der französische Lieteraturgeschichtsschreibung markiert werden. Der englische Wortgebrauch macht diesen Unterschied nicht. Der allgemeine Ausdruck 'migration literature' bezieht sich auf alle Erscheinungen der Literatur, die mit Landesund Kulturwechsel einhergehen, unabhängig von ihrem politischen Hintergrund. ${ }^{3}$ Diese Kategorisierung hat den offensichtlichen Vorteil, dass sie mehr Sinn für kleinere Abweichungen aufweist, und dass sie das Verwischen der politschen Gesichtspunkte ausschlie $\beta t$, wofür die ungarische Literaturgeschichtsschreibung anschauliche Beispiele liefert.

Als das Problem der Emigration als ein strukturbildendes Element der zeitgenössischen ungarischen Kultur das erste Mal erwähnt wurde, vertrat István Vas, einer der wichtigsten Dichter seiner Zeit, in einem Artikel mit dem Titel Ungarische Dichtung im Westen (1969), der in der Zeitung des Zentralkomitees der Sozialistischen Arbeiterpartei Ungarns erschienen ist, die Meinung ${ }^{4}$, dass es Emigrantenliteratur gar nicht gäjbe, weil die in West-Europa lebenden ungarischen Autoren einen regen Kontakt zu der literarischen Szene in Ungarn entwickeln könnten, ja sie könnten sogar ab und zu das Land besuchen. István Vas hat also aus den von John Neubauer aufgelisteten Aspekten nur einen berücksichtigt, und dabei die Lage verfälscht, denn die in der Emigration lebenden Autoren hatten am Ende der 60-er Jahre überhaupt keinen Zugang zur ungarischen Öffentlichkeit. Die ungarischen Behörden kontrollierten die Beziehungen zu ihnen sogar streng, außer wenn die Geheimdienste die Kontaktaufnahme befiehlen.

Einige Jahre vor dem Erscheinen des Artikels von Vas gab László Könnyü sein Werk Az amerikai magyar irodalom története szemelvényekkel (Die Geschichte der ungarischen Literatur in den USA, 1961) heraus, und fünf Jahre später erschien das Werk von Ferenc Mérő in der Bundesrepublik Deutschland Emigrációs magyar irodalom (Ungarische Emigrantenliteratur, 1966). Der Begriff der Emigration wurde in den relevanten Werken der 70-er Jahre nicht mehr verwendet. Auch die im westlichen Europa oder in den USA herausgegeben Bücher bildeten keine Ausnahme. Er wurde durch die ziemlich merkwürdige Adjektivkonstruktion 'westlich-ungarisch' ersetzt. Zur Kanonisierung dieser Adjektivkonstruktion kommt es im 4. Band des von der Ungarischen Akademie der Wissenschaften herausgegenen Standardwerks Die Geschichte der ungarischen Literatur zwischen 1945 und 1975 (1982). 
Das Beseitigen des Begriffs 'Emigration' und sein Ersetzen durch eine Adjektivkonstruktion hing offensichtlich damit zusammen, dass sein politischer Inhalt für die Ungarische Sozialistische Arbeiterpartei unakzeptabel war ${ }^{5}$, davon gar nicht zu sprechen, dass die Emigration im Bewusstsein der Zeitgenossen von den Erinnerungen an den Aufstand des Jahres 1956 und von dem darauf folgenden Ressentiments nicht zu trennen war. In der zweiten Hälfte der 60-er Jahre entstand auch in vielen Vetretern der westlichen Emigration ein merkwürdiges Verständnis für die Ziele des kommunistischen Regimes in Ungarn. Das Adjektiv 'westlich' bezeichnete damals keine Himmelsrichtung, viel mehr die andere, gegnerische Seite des kalten Krieges. Das Vertuschen des Begriffs 'Emigration' und sein Ersetzen durch die Adjektivkonstruktion 'westlich-ungarische' Literatur entsprach dem unehrlichen Program der kommunistischen Kulturpolitik, die das Regime auch durch die Verharmlosung der Konnotationen des Begriffs 'Emigration' legitimieren wollte. Diese Verharmlosung war eines der wichtigsten Merkmale der damaligen offiziellen Sprache.

Der Begriff der 'Emigration' kehrte nur langsam in den Wortgebrauch zurück. In dem 1986 erschienenen Werk von Miklós Béládi, Béla Pomogáts und László Rónay A nyugati-magyar irodalom 1945 után (Die westlich-ungarische Literatur nach 1945) wird er immer noch als ein Synonym der im Titel gefestigten Adjektivkonstruktion, letztendlich als ein sprachliches Element der damaligen opportunistischen, verfälschend gleichschaltenden Denkweise gebraucht, die das Erkennen von komplexen literarisch-politischen Phänomenen verunmöglicht hat. Die hier zitierte längere Passage lässt keinen Zweifel daran, dass die Darstellung der Emigrationsliteratur immer noch der sogenannten Emigrantenpolitik der Sozialistischen Arbeiterpartei Ungarns untergeordnet wurde: „Auf der politischen Palette der westlich-ungarischen Literatur (...) findet man recht unterschiedliche Ansichten. Die Vertreter der diversen ideologischen, politischen Auffassungen beurteilen die Ergebnisse der Entwicklungen in Ungarn ganz unterschiedlich. Der Rahmen der politischen Orientierung der Emigration, bzw. der westlich-ungarischen Literatur reicht von einer radikalen Opposition und Absonderung bis zu einer annehmbaren Kritik und der Suche nach den Möglichkeiten des gegenseitigen Verständnisses. Die ungarische Politik berücksichtigt diese Einstellungsunterschiede der ungarischen Öffentlichkeit im Westen, d.h. die Äußerungen der Vertreter der diversen Meinungsrichtungen über die Lage, über die Anstrengungen und Ergebnisse der ungarischen Gesellschaft. Die ungarische Regierung erwartet von den Autoren in der Emigration, die Entwicklungen in Ungarn mit einer bestimmten Loyalität wahrzunehmen. Diese Loyalität findet mit einer kritischen Haltung fraglos Übereinstimmung."6

Für die Rückkehr des Begriffs der 'Emigration' in den öffentlichen Diskurs und für den doppelte Wortgebrauch (Emigrantenliteratur/westlich-ungarische Literatur) liefern auch die Werke des in der Bundesrepublik lebendenden Historikers 
Gyula Borbándi wichtige Beispiele. ${ }^{7}$ Der doppelte Wortgebrauch blieb in Ungarn bis heute erhalten. Im dritten Band des umfassenden Handbuchs A magyar irodalom történetei (Die Geschichten der ungarischen Literatur, 2007) finden wir einen Beitrag von Móna Dániél und Tünde Varga über Die Zwischenräume der westlich-ungarischen Literatur und einen anderen Beitrag von Sándor Hites über Die Prosa der Emigration nach 1956. Einer der Herausgeber des Handbuchs, Mihály Szegedy-Maszák, vermied beide Wendungen, als er die Werke des in England geborenen Tibor Fischer erörterte, wobei er dessen Namen der ungarischen Konvention folgend als Fischer Tibor erwähnte. Die Vermeidung beider Wendungen ist ein klares Zeichen dafür, dass Mihály Szegedy-Maszák mit der Struktur des ungarischen literaturgeschichtlichen Bewusstseins unzufrieden war, aber keine Möglichkeiten für die Veränderung seiner Struktur sah. Er war der erste in der Reihe der ungarischen Literaturhistoriker, der die Literatur der Emigration in der Geschichte der gesammten ungarischen Literatur im 20. Jahrhundert für wesentlich, sogar essenziell hielt. „Im 20. Jahrhundert gewann die ausserhalb der Staatsgrenzen geschriebene Literatur eine grundlegende Bedeutung. Wegen der kulturellen Wiedervereinigung Europas, die der wirtschaftliche Vereinigung folgte, entstanden immer neue Werke in westlichen Ländern, die nicht durch die Sprache zu der ungarischen Literatur gehörten. Die Werke von im Ausland lebenden Autoren ungarischer Abstammung sind aus der Perspektive der Geschichte der ungarischen Literatur als eine Art Anhang zu betrachten."

Wie sieht die Struktur aus, die die Werke von Autoren wie z. B. George Tabori, Teréza Mora, Tibor Fischer, Lorand Gaspar, Agota Kristof, Melinda Nadj Abonji, Edith Bruck, George Szirtes, usw. nur als Anhänge betrachten kann? Diese Frage impliziert eine weitere Frage, die sich die ungarischen Literaturhistoriker immer wieder stellen müssen: Was ist das Ungarische an der ungarischen Literatur? Welche impliziten und expliziten Regel erschaffen die Grenzen und den Raum der ungarischen Literatur?

Das Konzept der Nationalliteratur - ihre Tradition erschien in einer gereiften Form im Werk von Sámuel Pápay 1808 A magyar literatúra esmérete (Die Kenntnis der ungarischen Literatur) -, beschränkte den Referenzraum der ungarischen Literatur von der Zeit der Reformation auf ungarisch geschriebene Werke, obwohl die ungarische Literatur, wie alle europäische Literaturen, zu jeder Zeit ziemlich reich an mehrsprachigen Autoren war. Das bis heute gültige Grundprinzip wurde von Ferenc Toldy 1865 deutlich gemacht: „Die gesammte oder die universelle Geschichte einer Nationalliteratur umfasst alle Bereiche einer Literatur, d.h. auch die wissenschaftlichen und die fremdsprachigen Werke; die national orientierte Literaturgeschichte behandelt im Gegensatz zur universellen nur diejenige Werke, die in der jeweiligen Nationalsprache geschrieben worden sind, und vollzieht dies in einem kausalen Zusammenhang, mit besonderer Aufmerksamkeit auf Werke, in denen sich die Eigentümlichkeiten des Nationalgeistes 
in besonderer Weise äußern." ${ }^{\prime 9}$ Ferenc Toldy bevorzugte das Konzept einer auf die Nationalsprache und auf die Äußerungen des Nationalgeistes gerichtete Nationalliteratur, und damit folgte er den Bedürfnissen seiner Zeit. Die ungarische Literaturgeschichte blieb aber diesem Rahmen bis heute treu, d.h. sie hat sich nie kritisch mit den Vorgaben auseinandergesetzt.

Würde man dieses Konzept auf die tschechische Literatur anwenden, würde es bedeuten, dass zum Beispiel die auf Französisch geschriebenen Werke von Milan Kundera nicht zu ihrem Grundstoff gehören, sondern nur als Teile eines Anhangs erwähnt werden könnten. Aus der Perspektive der tschechischen Literatur wäre es natürlich absurd, es müsste als eine sinnlose Selbstverstümmelung wahrgenommen werden. Weder die tschechische noch die slowakische Literaturgeschichte hat Konstruktionen wie etwa 'westlich-tschechisch' oder 'westlich-slowakisch' eingeführt. Sie benutzen viel mehr Begriffe wie 'literatura disidentú'oder 'exilová literatúra' ${ }^{10}$, die dem deutschen Konzpt der Exilliteratur entsprechen. Die Werke und die Autoren, die unter diesem Aspekt behandelt werden, gehören ohne Zweifel zum Kern der tschechischen und der slowakischen Literatur. ${ }^{11}$

Die Struktur der rumänischen Literaturgeschichtsschreibung beruht auf ähnlichen Prinzipien, wie die ungarische. Es stand sowohl für Eugen Lovinescu Mitte der 20-er Jahren des vorigen Jahrhunderts, als auch für George Călinescu, der seine Literaturgeschichte im Jahre 1941 veröffentlichte, außer Frage, dass die einzige Sprache der rumänischen Literatur das Rumänische sein kann. Călinescu hat sich zwar mit der Tatsache auseinandersetzen müssen, dass rumänische Autoren auch in Fremdsprachen schaffen, das hielt er aber für eine ,erklärbare”, aber „keineswegs preisenswürdige Entwicklung”" ${ }^{2}$. Später erlebte die rumänische Literatur die sehr ernste Provokation, dass Autoren, die einst auf Rumänisch geschrieben hatten, ihre Werke in der Emigration in Fremdsprachen, vor allem auf Französiche schufen, und damit europaweit berühmt wurden (z.B. Tristan Tzara, Eugène Ionesco, Mircea Eliade, Emil Cioran), und das selbe lässt sich über berühmte Autoren sagen, die zwar aus Rumänien stammten, aber nur selten oder nie Rumänisch schrieben (z.B. Paul Celan, Rose Ausländer, Oscar Pastior, Elie Wiesel, Herta Müller, Ádám Bodor, usw.). Es muss auch erwähnt werden, dass auffallend viele führende Experten der vergleichenden Literaturwissenschaft in den USA und in Frankreich aus Rumänien stammen (z.B. Matei Călinescu, Sorin Alexandrescu, Thomas Pavel, Virgil Nemoianu, Mihai Spăriosu, Marcel Cornis-Pope, Christian Moraru). Das literaturgeschichtliche Denken in Rumänien versucht Autoren, die mit ihren fremdsprachigen Werken Ruhm und Ansehen in Europa und in den USA erlangten, ohne die Veränderung des substanziellen Prinzips der Einsprachigkeit der rumänischen Literatur zu integrieren. Dieser Integrationsversuch bleibt also systemwidrig. ${ }^{13}$ Der nationale Stolz wird befriedigt, das althergebrachte Konzept der Einsprachigkeit und der sprachlichen Homogenität bleibt aber unantastbar. 
Zum Verständnis der heutigen Situation in Ungarn muss man auch die Tatsache berücksichtigen, dass das Grundkonzept der historischen Struktur der ungarischen Literatur nach dem ersten Weltkrieg durch eine neue Raumwahrnehmung zu ergänzen war. Die Hauptforderung nach der Einsprachigkeit als Merkmal der ungarischen Literatur wurde noch rigoroser eingefordert, es entstanden allerdings räumliche Substrukturen in denjenigen Gebieten, die an Rumänien, die Tschechoslowakei oder Jugoslavien abgetreten wurden (jeweils mit zahlenmäßig großer ungarischer Bevölkerung), und diese Substrukturen entwickelten ein eigenes literarisches Bewusstsein, d.h, es entstanden 'kleine Literaturen' (littérature mineur) innerhalb der vermeintlich homogen ungarischen Literatur. Diese 'kleinen Literaturen' verfügen über vielfältige Institutionen, über Lehrstühle an Universitäten, über literarische Presse und Verlage, und es gibt auch ein Leserpublikum mit besonderem Interesse an dieser regionalen Literatur. Diesen 'kleine Literaturen' an der Peripherie der sprachlich einheitlichen Nationalliteratur wies das literarische Bewusstsein des Zentrums eine besondere Funktion zu. Das von Ferenc Toldy 'Nationalgeist' genannte, abstrakte Phänomen, das die eigentliche, geschichtlich unveränderliche Essenz der Nationalliteratur sein sollte, drückte sich im Falle dieser regionalen Literaturen in der Zugehörigkeit zu der dem Vaterland abgekoppelten Heimat und im Bestehen auf der nationalen Identität und der moralischen Aufgabe des Behaltens der Muttersprache aus. Diese Grundhaltung wurde und wird auch heute von den Autoren, die in ungarischen Regionen außerhalb den Staatsgrenzen Ungarns leben, erwartet. Die meisten Städte bildeten vor dem ersten Weltkrieg eine sehr komplexe multiethnische Umgebung, die für die Gestaltung der eigenen Identitäten maßgeblich war. Die Spuren dieser multiethnischen Umgebungen findet man in den Werken von Mór Jókai, Kálmán Mikszáth, Gyula Krúdy, Sándor Márai und Dezső Kosztolányi. Die ungarische Literaturgeschichtsschreibung stellte allerdings die Forderung nach einer statischen und geschlossenen Nationalidentität an alle Autoren außerhalb der ungarischen Staatsgrenzen. Diese Forderung transportiert sehr direkte Wertvorstellungen, und sie übt eine moralische Kontrollfunktion über die Identität der Autoren aus.

Im Gegensatz zu den Literaturhistorikern in Ungarn, die die Geschichte der ungarischen Literaturen außerhalb Ungarns aufgrund einer Rhetorik der Beharrlichkeit und einer damit einhergehenden Opferrolle schrieben ${ }^{14}$, versuchten viele KollegInnen aus Rumänien, Serbien und aus der Slowakei dies zu vermeiden. Im Lexikon der ungarischen Literatur in der Tschecho/Slowakei 1918-2004 fehlen die Diskurselemente der Opferrhetorik gänzlich. ${ }^{15}$

Eine tiefgehende Kritik an den Erwartungen an die Rhetorik kommt zuerst im Werk von Imre Bori, A jugoszláviai magyar irodalom története (Die Geschichte der ungarischen Literatur in Jugoslawien, 1968), zum Ausdruck, in dem er einen eigenen Kontext für die ungarische Literatur in Serbien erstellt. Imre Bori versucht ein neues Konzept zu entwickeln, und beruft sich dabei auf die Perspektive 
von Oszkár Jászi, die in der ungarischen Kultur nach dem ersten Weltkrieg kaum beachtet wurde. Oszkár Jászi verfasste 1922 in seiner Wiener Emigration einen wichtigen Artikel mit dem Titel A magyar kultúra decentralizációja (Die Dezentralisation der ungarischen Kultur). Er war überzeugt, dass der Verlust des einheitlichen staatlichen Rahmens im Jahre 1920 sogar eine Chance zur Überwindung der zentralisierten Homogenität der ungarischen Kultur liefern könnte. Imre Bori zitiert den Artikel und stimmt Jászi zu: „Das geistige Leben der abgekoppelten ungarischen Bevölkerungsteile muss nicht mehr die Verpflanzung von Sämlingen aus Budapest sein, sondern es wird sich aus den lokalen Umständen des engeren Regionallebens ernähren und es wird daraus organisch wachsen."16

Die festgeschriebene, auf die Moral der Heldenhaftigkeit bauende Rhetorik diente als ein wichtiges Mittel im Interesse des Beibehaltens der hierarchischen und zentralisierten Struktur der ungarischen Kultur nach 1920. Gábor Sonkoly hat in seinem Werk Erdély városai a XVIII-XIX. században (Die Städte Transsylvaniens im 18.-19. Jahrhundert, 2001) bewiesen, dass sich die Beziehungen zwischen den lokalen Räumen und dem nationalen Raum Mitte des 19. Jahrhunderts, als der nationale Raum entstand, nicht unbedingt hierarchisch aufbauten. Die städtischen Patriotismen waren nicht unbedingt die lokalen Repräsentanten der nationalen Heimat. ${ }^{17}$ Levente T. Szabó betont aber, dass in der Herausbildung der einheitlichen Literatursprache, die eine ganz wesentliche Rolle im Entstehen der Nation spielte, die hierarchisierende Denkweise stark zur Geltung gekommen war. Die nationalen Vorstellungen beruhen nie auf einer konsensualen Stabilität, sie stellen sich eher als ein Terrain dar, in dem „Konsens und Einwilligungen miteinander kämpfen". ${ }^{18}$

Die ungarischen Literaturen in Rumänien, Serbien und in der Slowakei definierten sich im Rahmen einer hierarchischen Struktur aus der Perspektive des Zentrums durch die Zugehörigkeit zum heimatlichen Raum und das Bestehen auf der Muttersprache innerhalb einer fremdsprachigen Umgebung. Die Emigrantenliteraturen waren viel mehr durch das Fehlen von Zugehörigkeit zu irgendeinem vorhandenen Raum und eine verzehrende nostalgische Sehnsucht nach der verlassenen Heimat bestimmt. Miklós Béládi, Béla Pomogáts und László Rónay stellen die existenzielle Situation der Emigrantenautoren folgender Weise dar: „Die Emigrantenautoren schaffen in einer fremden Umgebung, für die es gar nicht wichtig ist, was ein Bürger des Landes auf ein Blatt Papier auf einer völlig unbekannten Sprache schreibt. [...] Die ungarische Literatur im Westen ist in Wirklichkeit eine unvollständige Literatur, sie leidet unter Mängeln, auch wenn der Autor die Bedenken und die Ängste, die auf schriftstellerische Einsamkeit zurückzuführen sind, überwältigen kann. [...] Die Schriftsteller der Diaspora schaffen einsam. Sie müssen sich zu der Treue zur Muttersprache, zu der ungarischen Literatur in einem fremden Umfeld bekennen, und das lastet oft so drückend auf ihnen, dass es am Ende zum Verstummen, zur totalen Erschöpfung führt." ${ }^{19}$ Die 
Hauptmotive der allgemeinen Beschreibung der Situation wiederholen sich tautologisch, wenn die einzelnen Autoren auf die Bühne treten. In den Werken der Emigration geht es immer um die Wahrnehmung des Mangels. László Cs. Szabó mag sich wohl in seinen Gedichten über die geistigen Anstrengungen der Emigration, über die unüberwindbaren Mängel und die Disposition der dauerhaften Trennung offen aussprechen., ${ }^{20}$ Im Falle von Sándor Márai wird eher das Motiv der Treue zur ungarischen Sprache und zum Nationalgeist hervorgehoben: „Was verbindet Márai mit dem Magyarentum? Diese Frage müssen wir auch deshalb stellen, weil er sein Befremden über das heutige Ungarn in seinen Tagebüchern immer wieder provokativ äußert. Er legt aber ein Bekenntnis für die unsterblichen Werke der ungarischen Literatur, für Autoren, deren Schriften und Personen er auch in der Emigration so anschaulich zitiert hat, ab. Er war in der ungarischen Kultur und Sprache tief verwurzelt. In einer seiner letzten Aufzeichnungen formulierte er diesen Gedanken mit verbindlicher Klarheit: „Die Muttersprache ist die einzige Heimat." 21

Die Wirkung dieser Lesereinstellung blieb in Ungarn bis heute bestehen. Die homogenisierende Rezeption der ungarischen Emigrantenliteratur, deren Vielfältigkeit und Dispersität im Rahmen der bestehenden Struktur nicht veranschaulicht werden kann, kann auch dadurch nicht behoben werden, dass man ihre Interpretation ins Begriffssystem der 'migration literature' einfügt, obwohl man dadurch einen offenen Zugang zu einer Reihe von bislang unerforschten kulturellen Beziehungen und Werken hätte. Aber auch dieses Begriffssystem lässt nur die auf Transgressionen, Hybridität und transnationale Bewegungen bezogenen Erfahrungen zum Vorschein kommen. Das Problem liegt eher im Wesen der Nationalliterarur, in ihrem Erwartungshorizont und in ihrer Raumstruktur. Um es zu erkennen und zu beheben, muss man sie kritisch umgestalten. Bei der kritischen Umgestaltung kann uns das Problem der Emigration durchaus behilflich sein. Während Béládi, Pomogáts und Rónay in den Gedichten von László Kemenes Gréfin, der seit 1977 in Montreal lebt, nur die Nostalgie und eine 'aristokratische Einsamkeit' wahrnehmen, „die oft zu skeptischen Interpretationen führt"'22, bemerken Móna Dánél und Tünde Varga in ihrer Erörterung über Kemes Gréfin, dass ihm „die Situation der westlich-ungarischen Literatur die Möglichkeit für die Umdeutung der ungarischen Nationalliteratur anbietet"23. Dávid Szolláth bemerkt in ähnlicher Weise, dass die Bedeutung der poetischen Reflexion im Roman von Miklós Mészöly, Der Tod des Athleten, dem in Paris lebenden Pál Albert in seiner 1966 geschriebenen Kritik aufgefallen ist, und er den Roman deshalb zusammen mit den damals erschienenen Werken der neuen französischen Romankunst, die damals in Ungarn nocht nicht bekannt waren, erwähnen konnte, weil er die ungarische Literatur synchron mit der französischen Literatur gelesen hat. ${ }^{24}$ 
Den Ausgangspunkt der Neugestaltung und des Wettkampfes zwischen den Konsensen könnte - im Einklang mit Imre Bori - der Artikel von Oszkár Jászi Die Dezentralisation der ungarischen Kultur bilden. Der Artikel erschien im serbischen Srenjanin (Nagybecskerek) in der Zeitschrift Fáklya 1922. Die Zeitschrift wurde von János Somfai gegründet, der nach der Räterepublik in Ungarn zu einer Gefängnisstrafe verurteilt wurde. 1920 wurde der Strafvollzug gelockert, und er floh aus dem Internierungslager nach Wien, wo er den Kontakt wahrscheinlich auch mit Oszkár Jászi aufgenommen hat. In Wien arbeitete er als Journalist für die Tageszeitung Bécsi Magyar Újság. Im Jahre 1921 kehrte er doch nach Srenjanin zurück, wo er seine eigene Zeitschrift mit dem Titel Fáklya gründete, die aber nur vier Monate bestand.

Jászi träumte von einer kulturellen Diversität, die ,nicht mehr als eine Diktatur von Budapest über die Peripherie, über das Land funktionieren wird, sondern es entsteht ein Wettkampf und ein gegenseitiger Wechsel zwischen selbständigen, lebendigen, freien und gleichrangigen lokalen Kulturen, die aus dem eignenen Boden wachsen, und all das wird der Entwicklung der gesammten ungarischen Kultur dienen." 25

Die Nationalliteratur muss sich jeder Zeit und im jedem Aspekt der Kultur, d.h. im Diskurs der Poetik, der Ideologie und der Politik mit heterogenen Bewegungen und Deterritorisationsversuchen auseinandersetzen. Die bis heute konsensuale Auffassung der Nationalliteratur bestimmt ein funktioneller Zwang. Die Elemente, die eine Provokation für die Homogenität bedeuten, werden entweder in bipolaren Gegensätzen eingeordnet, und dadurch vom Zentrum ferngehalten, oder sie werden vertuscht und ausgeschlossen, wie man im Falle der in Fremdsprachen geschriebenen Werke der ungarischen Literatur sieht.

Was für eine Territorisierung könnte den Wettkampf und den gegenseitigen Wechsel zwischen den freien und gleichrangigen lokalen Kulturen sichern? Das Konzept dieser Raumordung könnte auf der Erfahrung der Ähnlichkeiten entworfen werden. Das Ähnlichkeitsdenken, das in der Kuturtheorie von Anil Bhatti ins Zentrum tritt ${ }^{26}$, entsteht aus der Skepsis gegenüber den dialogischen, die Differenz letztendlich stabilisierenden Modellen, und es lässt sich als Topologie von Heterogenitäten, d.h. von polizentrischen Handelsräumen und von mehrsprachigen, plurikulturellen Gesellschaften verstehen. „Zusammenfassend lässt sich festhalten, dass die theoretische und praktische Profilierung von Ähnlichkeit zeigt, inwieweit es in plurikulturellen Gesellschaften eher darum geht, eine gesellschaftliche Umgangspraxis mit komplexer Diversität zu fördern, als um die Konstruktion von geschlossenen Kulturen, die durch Repräsentanten verhandlungsfähig gemacht werden. Es geht darum, das Approximative in Prozessen der Annäherung - und auch der Distanzierung - zu beschreiben. Statt Identitäten wären Asymptoten zu beschreiben." ${ }^{27}$ In diesem Konzept werden die kulturellen 
und gesellschaflichen Konstruktionen nicht aus der Phänomenalisierung der Dichotomie und der Differenz, d.h. nicht aus der Wahrnehmung von Grenzen und Epochenschwellen entwickelt, sondern viel mehr aus den Übergängen und Überlappungen, die für eine offene Konstellation sorgen.

Innerhalb der Struktur der ungarischen Nationalliteratur verdient die Emigration eine besondere Aufmerksamkeit, weil sie es notwendig macht, dass wir über die ungarische Literatur in Überlappungen mit anderen Literaturen denken. Wenn wir über Überlappungen sprechen, müssen wir in die Problematik auch die Theorie des Transnationalismus einbeziehen. In der ungarischen Fachliteratur wird diese Theorie auf die Fälle der geographischen und politischen Grenzübergänge beschränkt, die die mentale Einprägung der Grenzen als Reflexionsobjekte immer voraussetzen. ${ }^{28}$ Die Grenzphänomene verbinden sich mit den Vorstellungen der Differenzen, bleiben dabei aber die Kulminationspunkte der Macht und der Kontrolle. Die Theorie des Transnationalismus kann aber auch dabei helfen, dass die Vorstellungen der Nation aus dem Bereich der homogenisierenden Aspirationen der Macht entfernt werden, damit die Heterogenität des von der Macht territorisierten Raumes, in diesem Fall des literarischen Raumes, klar gezeigt werden kann. Die literarischen Räume von Ost-Mittel-Europa hatten schon immer eine mehrsprachige und plurikulturelle Ausdehnung, erst nach dem ersten Weltkrieg wurden die Differenzen im Interresse der Homogenisierung der Machtbereichen als Gegensätze instrumentalisiert. Das Aufgreifen des Problems der Emigration macht es möglich, diese räumliche Struktur neuzugestalten, weil die Emigraton nicht einmal als eine peripherische Erscheinung am Konzept der ungarischen Nationalliteratur teil hat. Wie schon das Beispiel von Kundera im Zusammenhang mit seiner tschechischen Herkunft so anschaulich gezeigt hat, ist die Behandlung der Emigration in der ungarsichen Literaturgeschichte nicht nur an sich absurd, sie macht auch die allgemeine Unhaltbarkeit der Raumwahrnehmung deutlich. Die Emigration kann nicht zuletzt auch die Kräfte des inneren Zusammenhalts im Raum der Nationalliteratur verstärken, denn sie lässt das Aufheben der lokalen Kontexte und die Beseitigung der Differenzen nicht zu, die als Voraussetzungen von Ähnlichkeiten immer ins Spiel gebracht werden.

Worüber ich hier spreche, ist also eine Hypothese für eine mögliche Selbstkorrektur. Auch diese Hypothese muss ihre Begrifflichkeit der Kritik aussetzen. Wenn sich aber die Raumstruktur der ungarischen Nationalliteratur als Modell einmal neugestaltet, wird die Verwendung des Begriffs der Emigration ihre Gültigkeit völlig verlieren. 


\section{Quellen}

Miklós Béládi, Béla Pomogáts, László Rónay, A nyugati magyar irodalom 1945 után, Gondolat, Budapest 1986.

Homi K. Bhabha (ed.), Nation and Narration, Routledge, London and New York, 1990.

Anil Bhatti Dorothee Kimmich, ,Albrecht Koschorke, et al.,„Ähnlichkeit. Ein kulturtheoretisches Paradigma”, in: Internationales Archiv für Sozialgeschichte der deutschen Literatur 36/1, 2011, S. 233-247.

Imre Bori, A jugoszláviai magyar irodalom története, Fórum, Novi Sad 1968

Václav Burian, Josef Galík, Lubomír Machala, Martin Podivínský, Jan Schneider, Česká a slovenská literatura v exilu a samizdatu, Hanácké noviny, Olomouc 1991.

Craig Calhoun, Nationalism, University of Minnesota Press, Minneapolis, 1997.

Thomas Hylland Eriksen, The Globalization, Bloomsbury, London, New York, 2014. 99-116.

Zoltán Fónod (Hrsg.), A cseh/szlovákiai magyar irodalom lexikona 1918-2004, Madách-Posonium, Preßburg 2004

Oszkár Jászi, A magyar kultúra decentralizációja, Fáklya, 1922. 1. 28.

Peter Leese, Carly McLaughlin, Władisłav Witalsz (ed.) Migration, Narration, Identity, Peter Lang Edition, Frankfurt am Main 2012.

Peter Morgan, Literary Transnationalism: A Europeanist's Perspective, Journal of European Studies, March 2017, Vol.47(1), S.3-20

John Neubauer, Borbála Zsuzsanna Török (Hrsg.), The Exile and Return of Writers from EastCentral Europe, Walter de Gruyter, Berlin, New York 2009.

Gábor Sonkoly, Erdély városai a XVIII-XIX. században, L'Harmattan - Atelier, Budapest 2001.

Mihály Szegedy-Maszák, A magyarság (nyelven túli) emléke, in: Mihály Szegedy-Maszák, András Veres, (Hrsg.), A magyar irodalom történetei III., Gondolat Kiadó, Budapest 2007.

Andrei Teiran, Romanian literature for the world: a matter of property, World Literature Studies, 2015, 7, S. 3-14.

Ferenc Toldy, A magyar nemzeti irodalom története, Szépirodalmi Könyvkiadó, Budapest 1987, S. 7-32.

Levente T. Szabó, A tér képei: tér, irodalom, társadalom, Komp-Press, Klausenburg 2008

\section{Anmerkungen}

1 „To become a migrant is to enter into a life of continual jolts and tremors, a life of uncertainty, but willingly or not it is also to act out hope and a belief in the future. Every migrant is to some degree embattled, enclosed, and at the same time exposed. Every migrant is also able to look, to see in a way that is not available to a traveller or a resident who has only remained within the nearly invisible borders of a single culture." Peter Leese, Introduction, in: Migration, Narration, Identity, Peter Leese, Carly McLaughlin, Władisłav Witalsz (ed.), Peter Lang Edition, Frankfurt am Main 2012, S. 8-9.

2 John Neubauer, Borbála Zsuzsanna Török (Hrsg.), The Exile and Return of Writers from East-Central Europe, Walter de Gruyter, Berlin, New York 2009, S. 9.

3 Es wird im Englischen auch das Wort 'dissident' verwendet, es bezieht sich aber nicht unbedingt auf einen Landeswechsel, es kann auch eine Art von innerer Emigration bedeuten.

4 István Vas, Költők között. Magyar költészet Nyugaton. Népszabadság 21.09. 1969, S. 12.

5 „Der Begriff 'Emigrant' drückt eine politische Kategorie, eine Unterscheidung, eine Art von Separatismus und Opposition aus," so Miklós Béládi, Béla Pomogáts, László Rónay, A nyugati magyar irodalom 1945 után, Gondolat, Budapest 1986, S. 15. 
6 Béládi, Pomogáts, Rónay, S. 7-8.

7 Er publizierte 1986 in Zürich eine Antologie mit dem Titel „Nyugati magyar esszéirók” (Ungarische Essayautoren im Westen), und in selben Jahr publizierte er ein weiteres Werk mit dem Titel „A magyar emigráció életrajza 1945-1985” (Die Biographie der ungarischen Emigration 1945-1985). Beide Werke wurden von dem Verband Európai Magyar Protestáns Egyesület herausgegeben. Der Verband wurde im Jahre 1969 von protestantischen Emigranten, die Ungarn nach dem gescheiterten Aufstand 1956 verlassen haben, in Basel gegründet. Der zweite Band von Borbándi wurde in Ungarn knapp vor der Wende, 1989, herausgegeben.

8 Mihály Szegedy-Maszák, A magyarság (nyelven túli) emléke, in: Mihály Szegedy-Maszák, András Veres, (Hrsg.), A magyar irodalom történetei III., Gondolat Kiadó, Budapest 2007, S. 831.

9 Ferenc Toldy, A magyar nemzeti irodalom története, Szépirodalmi Könyvkiadó, Budapest 1987, S. 24.

10 An diesem Punkt danke ich László Szigeti für seine wertvollen Hinweise.

11 Siehe Václav Burian, Josef Galík, Lubomír Machala, Martin Podivínský, Jan Schneider, Česká a slovenská literatura v exilu a samizdatu, Hanácké noviny, Olomouc 1991.

12 George Călinescu, History of Romanian Literature. Milan: UNESCO - Nagard 1988, S. 840 .

13 Siehe dazu Andrei Teiran, Romanian literature for the world: a matter of property, World Literature Studies, 2015, 7, S. 3-14.

14 Ein markantes Beispiel für diesen Diskurs liefert Béla Pomogáts in seinem Werk ,,Jelenidő az erdélyi magyar irodalomban" (Gegenwart in der ungarischen Literatur von Transsylvanien). Im Vorwort lesen wir: „Die Entstehung und das Bestehen von ungarischen Literaturen außerhalb Ungars, nicht zuletzt die der ungarischen Literatur in Rumänien ermöglichte eine opfervolle organisatorische Arbeit, eine Reihe von heldenhaften Anstrengungen." (Pomogáts Béla, Jelenidő az erdélyi magyar irodalomban, Magvetö, Budapest 1987, S. 5.) Am Ende seines Buches wendet sich Béla Pomogáts an den jungen Autoren dieser Literatur in folgender Weise: „In dieser Situation müssen die Vertreter der literarischen Kultur eine sehr große Verantwortung übernehmen. Es ist die Aufgabe der Mitglieder der jüngeren Generation, die leeren Posten der Literatur der ungarischen Nationalität zu besetzen. Sie müssen das durch so viel Opfer und durch so viele Geistesanstrengungen errichtete Gebäude dieser Lteratur standfester machen." (Pomogáts, S. 241.) Die selbe Rhetorik erscheint im Text eines Angehörigen der literarischen Gruppe „Elöretolt Helyőrség” (Vorgestossene Warte) aus Klausenburg, die heutzutage ein ganz wichtiger Akteur der offiziellen Kulturpolitik in Ungarn geworden ist. Siehe: Mihály Lakatos, „Csináljátok úgy, ahogy akarjátok.” És mi csináltuk, Tiszatáj, 1996, 10, S. 22.

15 Zoltán Fónod (Hrsg.), A cseh/szlovákiai magyar irodalom lexikona 1918-2004, Madách-Posonium, Preßburg 2004, S. 249-265.

16 Siehe: Imre Bori, A jugoszláviai magyar irodalom története, Fórum, Novi Sad 1968, S. 71.

17 Gábor Sonkoly, Erdély városai a XVIII-XIX. században, L'Harmattan - Atelier, Budapest 2001, S. 160-163.

18 Levente T. Szabó, A tér képei: tér, irodalom, társadalom, Komp-Press, Klausenburg 2008, S. 16.

19 Miklós Béládi, Béla Pomogáts, László Rónay,. S. 18.

20 Ebenda, S. 108.

21 Ebenda, S. 96.

22 Ebenda, S. 247.

23 Móna Dánél, Tünde Varga, A nyugati magyar irodalom »köztes tere«, in: Szegedy-Maszák Mihály, Veres András, A magyar irodalom történetei III., Gondolat, Budapest 2007, S. 749. 
24 Dávid Szolláth, Olvasásmódok ütközése. Mészöly Miklós: Az atléta halála, In: Pontos észrevételek. Mészöly Miklóstól Nádas Péterig és vissza. Sensus füzetek. Jelenkor Kiadó, Budapest 2015, S. 14.

25 Oszkár Jászi, A magyar kultúra decentralizációja, Fáklya, 1922. 1. 28.

26 Anil Bhatti Dorothee Kimmich, ,Albrecht Koschorke, et al. „Ähnlichkeit. Ein kulturtheoretisches Paradigma", in: Internationales Archiv für Sozialgeschichte der deutschen Literatur 36/1, 2011, S. 233-247.

27 Ebenda, S. 245.

28 Siehe: Tímea Jablonczay, Transznacionalizmus a gyakorlatban: migrációs praxisok a könyvek, az írásmódok, a műfajok és a fordítási stratégiák geográfiájában, Helikon 2015, 2, S. 138.

Open Access. This is an open-access article distributed under the terms of the Creative Commons Attribution 4.0 International License (https://creativecommons.org/licenses/by/4.0), which permits unrestricted use, distribution, and reproduction in any medium, provided the original author and source are credited, a link to the CC License is provided, and changes - if any - are indicated. (SID_1) 
